\title{
A Loss Network Model with Overflow for Capacity Planning of a Neonatal Unit
}

\author{
Md Asaduzzaman · Thierry J. Chaussalet · Nicola \\ J. Robertson
}

Received: date / Accepted: date

\begin{abstract}
The main aim of this paper is to derive a solution to the capacity problem faced by many perinatal networks in the United Kingdom. We propose a queueing model to determine the number of cots at all care units for any desired overflow and rejection probability in a neonatal unit. The model formulation is developed, being motivated by overflow models in telecommunication systems. Exact expressions for the overflow and rejection probabilities are derived. The model is then applied to a neonatal unit of a perinatal network in the UK.
\end{abstract}

Keywords Neonatal unit · Overflow probability · Rejection probability · Queueing network · OR in health care

\section{Introduction}

In the UK, every year approximately $8-13 \%$ babies are born premature, very sick, or very small (RCPCH, 2007). These babies require some form of specialist support at birth and are admitted to a specialist neonatal unit for care. Many of them (1-3\% of total births) are extremely premature (gestational age $<27$ weeks) or have a very low birth weight $(<1000$ gms), and require neonatal intensive care (DH, 2005; RCPCH, 2007). Most hospitals are regularly unable to meet demand for specialist neonatal care in the UK (Parmanum et al, 2000). BLISS the premature baby charity reported that about $78 \%$ of all neonatal units in the UK had to close to new admissions at least once in the last six months (BLISS, 2005). The problem is exacerbated by several factors. First, the percentage of rejected admissions is increasing across neonatal units in the UK. Secondly, these rejections create risk and expensive transfer of sick babies. Thirdly, cots in neonatal units are very expensive; management wants to keep them highly utilised, which increases the rejection probability.

Md Asaduzzaman · Thierry J. Chaussalet Health and Social Care Modelling Group (HSCMG) School of Informatics, University of Westminster 115 New Cavendish Street, London W1W 6UW, UK E-mail: asaduzm@wmin.ac.uk

Nicola J. Robertson

UCL Elizabeth Garrett Institute for Women's Health Maple House, University College London

149 Tottenham Court Road, London W1T 7DN, UK 
In the UK, care for vulnerable babies is organised in a regional setting. Neonatal units are part of a neonatal/perinatal network; where a perinatal network consists of several units and aims to care for all sick newborns in that region. Infants of low gestational age $(<27$ weeks) or very low birth weight $(<1000$ gms) or requiring neonatal surgery have to be admitted to the neonatal intensive care unit of the perinatal centre within that region. A perinatal centre is a neonatal unit, which provides all levels of care: intensive care (ICU), high dependency (HDU) and special care (SCU). It is also known as the lead centre or the tertiary referral centre of a perinatal network and plays a vital role by providing the highest level of care for the most vulnerable babies. A perinatal centre has also some transitional care cots which may be used to handle overflow from special care depending upon demand and circumstances. Babies admitted at ICU are normally moved to HDU depending upon improvement and then to SCU from where they are allowed to go home or to a normal ward. But since cots in all three levels are scarce and in high demand, babies admitted from outside the network are often discharged from ICU and HDU and transferred back to the unit where they come from. Therefore, discharge from any unit is possible. An important characteristic of the neonatal unit is that it does not allow queues to form when all cots are occupied. In such cases, a baby is transferred to another neonatal unit. These transfers may adversely impact the health status of the baby due to extended transportation time and lack of immediate care. All these factors add to the challenge of planning and managing capacity of a neonatal unit.

Queueing theory and simulation are among the most popular and suitable healthcare modelling techniques when the system considered is heavily stochastic. Patient flow was analysed across the hospital by Koizumi et al (2005), Chaussalet et al (2006) and Jiang and Giachetti (2008) using queueing networks, and by Ferreira et al (2008) using simulation. Focussing on intensive care units, Ridge et al (1998) and Kim et al (1999) used simulation for bed capacity planning, while Griffiths et al (2006) proposed a queuing model for a similar problem. Simulation models often appeal because of their versatility and the user-friendly graphical interface of specialist simulation software, but may have drawbacks such as difficulties with interpretation of the results and validation, and high resource requirements (runtime, software cost, etc.). Queueing models are transparent mathematical models, but may require restrictive and sometime unrealistic assumptions to be solved analytically. When closed form solutions can be derived, queueing models can be easily implemented in a spreadsheet environment to obtain immediate estimates of quantities such as the number of beds required for given levels of service and immediate answers to 'what if' scenarios.

Among recent studies Kortbeek and van Dijk (2007) proposed an $M / M / c / 0$ loss model for capacity management in an Operating Theatre-Intensive Care Unit (OT-ICU) and Litvak et al (2008) developed an overflow model for capacity planning in intensive care. However, these models cannot be applied to this study for various reasons. The model proposed by Kortbeek and van Dijk (2007) cannot capture the overflow to transitional care as it happens in the neonatal case. The model by Litvak et al (2008) considered three classes of patients from where only a single class can be overflowed. Moreover, both papers used an approximation rather than an exact method to estimate the rejection probability. Asaduzzaman and Chaussalet (2008) proposed a loss network framework to capture the patient flow in a neonatal unit but they did not consider the overflow issue and the role of transitional care.

The main aim of this paper is to develop a model which can assist planners at perinatal centres in the UK with determining how many cots are required at all units of care to achieve desired levels of service in terms of probability of admission refusal and overflow to temporary care. The model is developed for the neonatal unit at University College London Hospital (UCLH), the perinatal centre of the North Central London Perinatal Network 
(NCLPN) and one of the busiest perinatal centres across the country. The proposed model is an overflow loss network model inspired from telecommunications systems (e.g., Kelly, 1991; van Dijk, 1993). Call blocking is a key performance measure in telecommunication networks (Abdalla and Boucherie, 2002). Systems in telecommunication with overflow traffic have also been widely used (Głąbowski et al, 2008). Boucherie and Mandjes (1998) derived a closed form solution of the equilibrium distribution to measure performance of cellular mobile communications networks. Sendfeld (2008) developed an open queueing network consisting of two queues and having some overflow capability from the first to the second queue. Our proposed model is based on the blocking and overflow mechanism as occur in telecommunication networks. The probability that a baby will overflow from the main unit to temporary care and the probability that a baby will be rejected from any unit have been derived by an exact rather than an approximation method.

The paper is organised as follows. In the next section we describe the structure of a perinatal centre and the flow of babies through its units. Data and preliminary results are described in section 3. Section 4 provides a loss network model with overflow, analysis of the model and the method for estimating the overflow and rejection probabilities. Section 5 provides the results obtained for the UCLH perinatal centre. Conclusions and further research direction are given in section 6 .

\section{Patient flow in a perinatal centre}

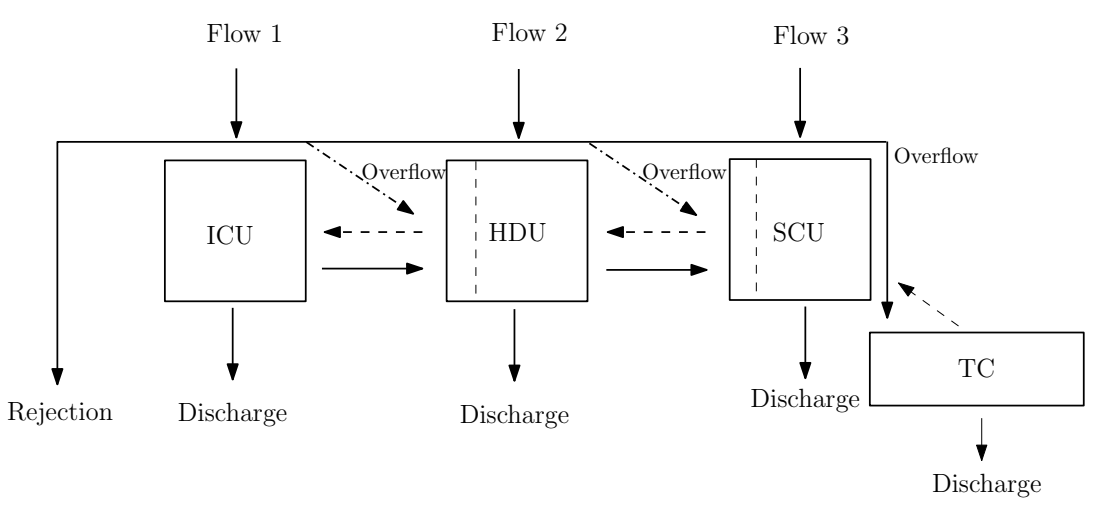

Fig. 1 Flow of babies in a perinatal centre

Most babies in a perinatal centre are admitted from the region served by the network. However, a substantial number of requests for admission come from outside the network. After delivery, babies are admitted to ICU, HDU, or SCU according to the severity of their condition, which is usually driven by their gestational age and birth weight. As their condition improves, babies will eventually be transferred to a unit providing a lower level of care (i.e. from ICU to HDU or HDU to SCU) within the perinatal centre or in another neonatal unit from where they are discharged. Similarly those admitted to HDU move to SCU before discharge. A perinatal centre has transitional care (TC) cots, which are used to provide temporary special care until an SCU cot becomes available. ICU (respectively HDU) babies are sometimes initially cared at HDU (respectively SCU) when all ICU (respectively 
HDU) cots are occupied. This temporary care is provided by staffing a cot with appropriate staff and equipment resources. This only occurs when babies must be admitted because they are booked at the same hospital or severely ill. Rejection from ICU (respectively HDU and SCU) occurs only when all cots at the ICU (respectively HDU and SCU) and HDU (respectively SCU and TC) are occupied.

Figure 1 schematically depicts patient flows for the three main units of a perinatal centre. Flow 1, 2 and 3 corresponds to requests for admission to ICU, HDU and SCU respectively.

\section{Case study: the UCLH perinatal centre}

At UCLH, perinatal centre of the NCLPN, intensive and high dependency care units have been merged for the purpose of better utilisation of cots. Therefore, UCLH has two units: neonatal intensive care unit (NICU) and special care baby unit (SCBU). In 2006, it had 12 NICU funded cots, 15 SCBU funded cots, and 8 TC cots. The data used for this study concern the UCLH perinatal centre and were collected through the South England Neonatal Database (SEND) and the NCLPN Transfer Audit between 1 January and 31 December 2006. Agreement from the Caldicott Guardian was obtained and the data were anonymised. The SEND data provide comprehensive and accurate information about each baby's stay in NICU and SCBU, including dates and times of arrival and discharge, source and reason of admission, birth weight and gestational age, while the NCLPN transfer audit data include daily counts of all requests for admission. In 2006, 1002 babies were admitted (possibly more than once) to the neonatal unit at UCLH; $31 \%$ percent were admitted to NICU and the remaining to SCBU. Of the NICU admissions $18.7 \%$ had gestational age $<27$ weeks. The

Table 1 Summary statistics of inter-arrival times and length of stay (days) of babies at the UCLH perinatal centre

\begin{tabular}{lcrr}
\hline Summary statistics & No. of babies & Mean & SD \\
\hline NICU & & & \\
Inter-arrivals (in days) & 443 & 0.82 & 0.99 \\
LoS (in days) & 308 & 14.09 & 20.33 \\
\hline SCBU & & & \\
Inter-arrivals (in days) & 905 & 0.40 & 0.57 \\
LoS (in days) & 884 & 7.76 & 7.63 \\
\hline
\end{tabular}

number of admissions to UCLH during the period was 299 for NICU and 870 for SCBU while the number of rejected admission requests was 144 for NICU and 35 for SCBU. Therefore, the total number of requests for admission was 443 for NICU and 905 for SCBU. For the estimation length of stay (LoS) we considered all 308 NICU babies and 884 SCBU babies who had been cared for in 2006. Some of these babies were admitted to UCLH in 2005.

The average inter-arrival time is 0.82 days for NICU and 0.40 for SCBU. More detailed statistics on inter-arrival times and LoS are given in Table 1. The mean LoS for NICU is 14.09 days and 7.76 days for SCBU. The mean LoS of the babies admitted to NICU differs significantly from the babies admitted to SCBU. 


\section{Proposed model framework and analysis}

\subsection{An overflow model for UCLH}

Consider a perinatal centre with two main units $U_{1}$ (NICU) and $U_{2}$ (SCBU) with $c_{1}$ and $c_{2}$ cots respectively. If all $c_{1}$ cots are occupied at NICU $U_{1}$ babies are admitted to SCBU $U_{2}$ initially (overflow) if there is an empty cot, and rejected otherwise. Similarly babies arrive at $\mathrm{SCBU}$; if all $c_{2}$ cots are occupied they are moved to the TC (overflow) unit $U_{0}$ containing $c_{0}$ cots and rejected if all cots are occupied. Our goal is to estimate the proportion of rejected babies from each unit. Figure 2 shows a detailed flow of babies for the UCLH perinatal centre.

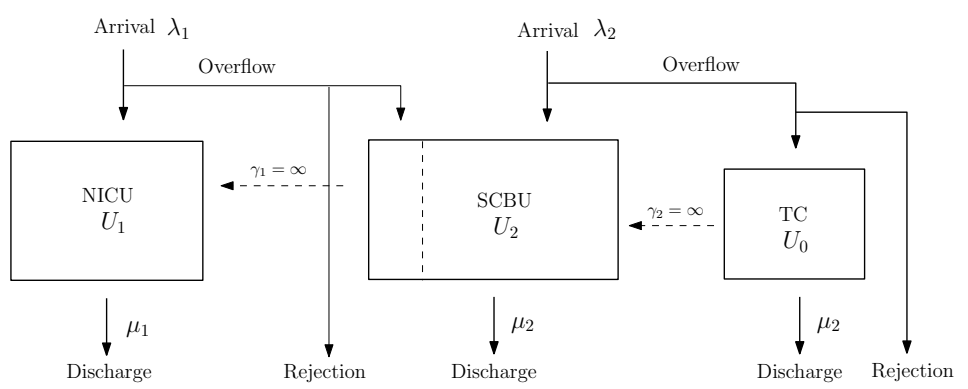

Fig. 2 An overflow model for the UCLH perinatal centre

\section{Modelling assumptions}

1. Requests for admission follow a Poisson process with rate $\lambda_{1}$ for NICU (respectively $\lambda_{2}$ for SCBU). The length of NICU care (respectively SCBU care) is exponentially distributed with mean $\mu_{1}^{-1}$ (respectively $\mu_{2}^{-1}$ ).

These assumptions make the model more tractable and are generally supported by the data, in particular for SCBU LoS and NICU arrivals, which have similar mean and standard deviations (see Table 1). Although for NICU LoS there are visible differences between mean and standard deviation, it should be noticed that in loss network models if the arrival process is Markovian the loss probability is insensitive to service time (LoS) distribution (e.g., Kelly, 1979; Davis et al, 1995; Erhardsson, 2001).

2. There is no waiting facility at any unit, implying a baby is either accepted or rejected. As discussed earlier, there is no waiting space for babies coming to be admitted at any unit. If all cots at the corresponding unit are occupied the baby is transferred elsewhere.

3. The flow from NICU to SCBU is not modelled explicitly. Since babies cared at NICU and discharged to SCBU get the same priority as new requests for admission at SCBU, discharges of babies to SCBU from NICU and new arrivals at SCBU are combined into a single Poisson arrival process.

This assumption enables us to make a simplified yet realistic description of the system, and to develop a more tractable model.

4. NICU babies who are in SCBU because of overflow from unit $U_{1}$ eventually join unit $U_{1}$ at rate $\gamma_{1}$. Similarly babies who are in TC eventually join unit $U_{2}$ at rate $\gamma_{2}$. 
Let $X_{i 0}(t)$ be the number of babies overflowing from unit $U_{i}, i=1,2$, moved to the corresponding overflow unit either $U_{2}$ or $U_{0}$ at time $t$, and denote $X_{i}(t)$ the number of babies at unit $U_{i}, i=1,2$, at time $t . \mathbf{X}=\left(X_{1}(t), X_{10}(t), X_{2}(t), X_{20}(t), t \geq 0\right)$ is a continuous-time Markov chain with state space given by

$$
\mathbf{S}=\left\{\mathbf{n}=\left(n_{1}, n_{10}, n_{2}, n_{20}\right): n_{1} \leq c_{1}, n_{10}+n_{2} \leq c_{2}, n_{20} \leq c_{0}\right\}
$$

where $n_{i}, i=1,2$, is the number of babies at the $i$ th main unit and $n_{i 0}, i=1,2$, is the number of babies at the $i$ th overflow unit. The transition rates $\mathbf{Q}=\left(q\left(\mathbf{n}, \mathbf{n}^{\prime}\right), \mathbf{n}, \mathbf{n}^{\prime} \in \mathbf{S}\right)$ are given by

$$
q\left(\mathbf{n}, \mathbf{n}^{\prime}\right)=\left\{\begin{array}{lll}
\lambda_{1} & \mathbf{n}^{\prime}=\mathbf{n}+\mathbf{I}_{1} \text { or } \mathbf{n}^{\prime}=\mathbf{n}+\mathbf{I}_{10}, & \text { if } n_{1}=c_{1} \\
\lambda_{2} & \mathbf{n}^{\prime}=\mathbf{n}+\mathbf{I}_{2} \text { or } \mathbf{n}^{\prime}=\mathbf{n}+\mathbf{I}_{20}, & \text { if } n_{10}+n_{2}=c_{2} \\
n_{i} \mu_{i} & \mathbf{n}^{\prime}=\mathbf{n}-\mathbf{I}_{i} \\
n_{i 0} \mu_{i} & \mathbf{n}^{\prime}=\mathbf{n}-\mathbf{I}_{i 0} \\
0 & \text { otherwise, }
\end{array}\right.
$$

where $\mathbf{I}_{i}$ and $\mathbf{I}_{i 0}$ denote $i$ th unit vectors. We are interested in deriving the overflow probability i.e. the probability that an admission request finds all NICU (or SCBU) cots occupied and the rejection probability i.e. the probability that a new arrival finds all cots occupied in both NICU and SCBU (or SCBU and TC).

Let $\pi(\mathbf{n})=\lim _{t \rightarrow \infty} P(\mathbf{X}(t)=\mathbf{n})$ denote the equilibrium distribution that there are $\mathbf{n}$ babies in all units. This equilibrium distribution of $\mathbf{X}$ is the unique distribution $\pi(\mathbf{n}), \mathbf{n} \in \mathbf{S}$ that satisfies the global balance equation,

$$
\begin{array}{r}
\lambda_{1}\left(\mathbf{1}_{\left\{n_{1}<c_{1}\right\}}(\mathbf{n})+\mathbf{1}_{\left\{n_{1}=c_{1}, n_{10}+n_{2}<c_{2}\right\}}(\mathbf{n})\right)+\lambda_{2}\left(\mathbf{1}_{\left\{n_{10}+n_{2}<c_{2}\right\}}(\mathbf{n})+\mathbf{1}_{\left\{n_{10}+n_{2}=c_{2}, n_{20}<c_{0}\right\}}(\mathbf{n})\right) \\
\left.+\sum_{i}^{2} n_{i} \mu_{i}+\sum_{i}^{2} n_{i 0} \mu_{i}\right] \cdot \pi(\mathbf{n})= \\
\lambda_{1}\left[\pi\left(\mathbf{n}-\mathbf{I}_{1}\right)+\pi\left(\mathbf{n}-\mathbf{I}_{10}\right) \mathbf{1}_{\left\{n_{1}=c_{1}, n_{10}+n_{2}<c_{2}\right\}}(\mathbf{n})\right] \\
+\lambda_{2}\left[\pi\left(\mathbf{n}-\mathbf{I}_{2}\right)+\pi\left(\mathbf{n}-\mathbf{I}_{20}\right) \mathbf{1}_{\left\{n_{10}+n_{2}=c_{2}, n_{20}<c_{0}\right\}}(\mathbf{n})\right] \\
+\sum_{i=1}^{2}\left(n_{i}+1\right) \mu_{i} \pi\left(\mathbf{n}+\mathbf{I}_{i}\right)+\sum_{i=1}^{2}\left(n_{i 0}+1\right) \mu_{i} \pi\left(\mathbf{n}+\mathbf{I}_{i 0}\right),
\end{array}
$$

normalised such that $\sum_{\mathbf{n} \in \mathbf{S}} \pi(\mathbf{n})=1$ and where $\mathbf{1}_{\{\cdot\}}$ denotes the indicator function of the event or set $\{\cdot\}$, i.e. $\mathbf{1}_{\{\cdot\}}(\mathbf{n})$ equals to 1 if $\mathbf{n} \in\{\cdot\} ; 0$ otherwise, for $\mathbf{n} \in \mathbf{S}$. The global balance equation expresses that the transition rate out of a state due to an arrival or discharge of a baby into the neonatal unit is equivalent to the transition rate into that state due to an arrival or discharge of a baby into the neonatal unit.

\subsection{Estimation of overflow and rejection probabilities}

The desired overflow and rejection probabilities can be computed from the equilibrium distribution of the system. 
A product form NICU-SCBU system

The local balance equations can be derived from the global balance equation (3),

$$
\lambda_{i}\left(\pi\left(\mathbf{n}-\mathbf{I}_{i}\right)+\pi\left(\mathbf{n}-\mathbf{I}_{i 0}\right)\right)=\left(n_{i} \mu_{i}+n_{i 0} \mu_{i}\right) \cdot \pi(\mathbf{n}) .
$$

Equation (4) has an explicit solution which is given by

$$
\pi(\mathbf{n})=G^{-1} \prod_{i=1}^{2} \frac{\rho_{i}^{\left(n_{i}+n_{i 0}\right)}}{\left(n_{i}+n_{i 0}\right) !}, \mathbf{n} \in \mathbf{S}, G=\sum_{\mathbf{n} \in \mathbf{S}} \prod_{i=1}^{2} \frac{\rho_{i}^{\left(n_{i}+n_{i 0}\right)}}{\left(n_{i}+n_{i 0}\right) !},
$$

where $\rho_{i}=\lambda_{i} / \mu_{i}$ is the traffic intensity at unit $U_{i}$. The proof follows from the observation that the equilibrium distribution (5) is the solution of local balance equations (4) and that any solution to local balance equations (4) must also be a solution to the global balance equation (3). This equilibrium distribution is a truncated multidimensional Poisson distribution from where overflow and rejection probabilities can be derived. The overflow probability for unit $U_{i}, i=1,2$, is then

$$
\begin{aligned}
O_{i} & =\sum_{\mathbf{n} \in T_{i}} \pi(\mathbf{n}) \\
& =\left(\sum_{\mathbf{n} \in T_{i}} \prod_{i=1}^{2} \frac{\rho_{i}^{\left(n_{i}+n_{i 0}\right)}}{\left(n_{i}+n_{i 0}\right) !}\right) /\left(\sum_{\mathbf{n} \in \mathbf{S}} \prod_{i=1}^{2} \frac{\rho_{i}^{\left(n_{i}+n_{i 0}\right)}}{\left(n_{i}+n_{i 0}\right) !}\right),
\end{aligned}
$$

where

$$
T_{1}=\left\{\mathbf{n} \in \mathbf{S} \mid\left(n_{1}=c_{1} \text { and } n_{10}+n_{2} \leq c_{2}\right)\right\},
$$

and

$$
T_{2}=\left\{\mathbf{n} \in \mathbf{S} \mid\left(n_{10}+n_{2}=c_{2} \text { and } n_{20} \leq c_{0}\right)\right\} .
$$

The rejection probability $R_{i}$ for unit $U_{i}, i=1,2$, can also be computed from equation (6) by substituting

$$
T_{1}=\left\{\mathbf{n} \in \mathbf{S} \mid\left(n_{1}=c_{1} \text { and } n_{10}+n_{2}=c_{2}\right)\right\},
$$

and

$$
T_{2}=\left\{\mathbf{n} \in \mathbf{S} \mid\left(n_{10}+n_{2}=c_{2} \text { and } n_{20}=c_{0}\right)\right\} .
$$

\section{Results}

We applied the model developed in the previous section to the case of UCLH. Validation and verification were done with staff at the UCLH perinatal centre. With the existing resources of 12 NICU, 15 SCBU and 8 TC cots, the rejection probability is 0.333 at NICU and 0.056 at SCBU (Table 2). The observed probability that an admission request will be rejected from NICU is 0.325 and 0.038 from SCBU. This shows that the model is accurate enough to estimate the rejection probabilities at each level of care in the UCLH perinatal centre and is an improvement over the standard loss network model developed previously by Asaduzzaman and Chaussalet (2008).

Results for different cot combinations with existing arrival patterns and LoS are derived and shown in Table 3 and 4. Table 3 shows that varying the number of TC cots has little effect on overflow and rejection probabilities at both NICU $\left(O_{1}, R_{1}\right)$ and $\operatorname{SCBU}\left(O_{2}, R_{2}\right)$. This confirms that the solution to the overflow and rejection problems in the neonatal unit is not to add more TC cots, but must be to increase cot capacity at SCBU and NICU. 
Table 2 Rejection probability- comparison between models

\begin{tabular}{lccc}
\hline Unit & Obs. rej. prob. & $\begin{array}{c}\text { Est. rej. prob. } \\
\text { (Standard model)* }\end{array}$ & $\begin{array}{c}\text { Est. rej. prob. } \\
\text { (Overflow model) }{ }^{* *}\end{array}$ \\
\hline NICU & 0.325 & 0.377 & 0.333 \\
SCBU & 0.038 & 0.070 & 0.056 \\
\hline *Standard loss network model with 12 NICU and 23 SCBU cots \\
**Overflow model in section 4 with 12 NICU, 15 SCBU, and 8 TC cots
\end{tabular}

Table 3 Overflow and rejection probabilities for 12 NICU, 15 SCBU and different TC cots at UCLH perinatal centre

\begin{tabular}{ccccccc}
\hline NICU & SCBU & TC & $O_{1}$ & $R_{1}$ & $O_{2}$ & $R_{2}$ \\
\hline $\mathbf{1 2}$ & $\mathbf{1 5}$ & 6 & 0.5153 & 0.3267 & 0.6252 & 0.0882 \\
& & 7 & 0.5053 & 0.3302 & 0.6520 & 0.0716 \\
& & $\mathbf{8}$ & $\mathbf{0 . 4 9 8 0}$ & $\mathbf{0 . 3 3 2 8}$ & $\mathbf{0 . 6 7 1 7}$ & $\mathbf{0 . 0 5 6 5}$ \\
& & 9 & 0.4927 & 0.3347 & 0.6859 & 0.0433 \\
& & 10 & 0.4889 & 0.3360 & 0.6961 & 0.0323 \\
\hline
\end{tabular}

Table 4 Overflow and rejection probabilities for different NICU and SCBU cot combinations with 8 TC cots

\begin{tabular}{ccccccc}
\hline NICU & SCBU & TC & $O_{1}$ & $R_{1}$ & $O_{2}$ & $R_{2}$ \\
\hline $\mathbf{1 2}$ & $\mathbf{1 5}$ & $\mathbf{8}$ & $\mathbf{0 . 4 9 8 0}$ & $\mathbf{0 . 3 3 2 8}$ & $\mathbf{0 . 6 7 1 7}$ & $\mathbf{0 . 0 5 6 5}$ \\
& 16 & & 0.5441 & 0.3138 & 0.5653 & 0.0389 \\
& 17 & & 0.5946 & 0.2915 & 0.4559 & 0.0256 \\
& 18 & & 0.6456 & 0.2671 & 0.3529 & 0.0161 \\
\hline 14 & 15 & 8 & 0.3734 & 0.2596 & 0.7346 & 0.0618 \\
& 16 & & 0.4083 & 0.2467 & 0.6429 & 0.0443 \\
& 17 & & 0.4488 & 0.2310 & 0.5433 & 0.0305 \\
& 18 & & 0.4927 & 0.2129 & 0.4427 & 0.0202 \\
\hline 16 & 15 & 8 & 0.2656 & 0.1901 & 0.7752 & 0.0652 \\
& 16 & & 0.2896 & 0.1815 & 0.6949 & 0.0479 \\
& 17 & & 0.3184 & 0.1707 & 0.6048 & 0.0340 \\
& 18 & & 0.3509 & 0.1581 & 0.5098 & 0.0233 \\
\hline 17 & 15 & 8 & 0.2187 & 0.1583 & 0.7895 & 0.0664 \\
& 16 & & 0.2379 & 0.1513 & 0.7137 & 0.0492 \\
& 17 & & 0.2612 & 0.1425 & 0.6275 & 0.0352 \\
& 18 & & 0.2880 & 0.1321 & 0.5355 & 0.0244 \\
\hline 18 & 15 & 8 & 0.1766 & 0.1292 & 0.8010 & 0.0674 \\
& 16 & & 0.1917 & 0.1236 & 0.7287 & 0.0502 \\
& 17 & & 0.2101 & 0.1165 & 0.6459 & 0.0363 \\
& 18 & & 0.2314 & 0.1080 & 0.5564 & 0.0254 \\
\hline
\end{tabular}

In Table 4 overflow and rejection probabilities are presented for different combinations of NICU and SCBU cots keeping the number of TC cots fixed at 8 . As the number of cots increases the rejection probability decreases. With 17 NICU, 16 SCBU and 8 TC cots, rejection probabilities are 0.1513 from NICU and 0.0492 from SCBU. With 18 NICU, 18 SCBU and 8 TC cots, $10 \%$ admission requests would be rejected from NICU and less than $3 \%$ from SCBU.

The NCLPN has the basic aim to provide all levels of care for $95 \%$ of mothers and babies within the network area where they live. The huge overflow and rejection levels at NICU derived from the model confirm the network is struggling to cope with demand with its current cot capacity. Furthermore the model reveals that capacity will have to increase significantly before the $95 \%$ target is achieved. 
Note however that increasing the number of cots at SCBU or TC can only reduce rejection of admissions but does not ensure quality of care. Therefore, while planning capacity allocation for a perinatal centre one should aim at keeping the expected overflow to SCBU from NICU for NICU babies and from SCBU to TC for SCBU babies to a minimum.

\section{Conclusion and further research}

All recent studies of neonatal care networks in the UK suggest that capacity is now a burning issue. It is claimed that capacity needs to be expanded immediately for most neonatal units in the UK. But the cost involved in running neonatal cots is very high because of the sophisticated equipment and highly skilled staff required. At NICU, one nurse is required per occupied cot. Therefore, expanding capacity can only be limited. On the other hand, transfer of babies between units due to capacity shortage is a major concern since it incurs risk and cost. Quality of care is another issue.

In this paper, we have developed a capacity planning tool based on loss queueing networks to study the impact of cot allocation to all care categories on admission rejection and overflow to temporary care. The work has been done in collaboration with the NCLPN where UCLH is the perinatal network centre. Network management reviews cot capacity once a year, including capacity of individual neonatal units based on measures such as the level of rejection and overflow of babies. Results showed that one in three admissions were rejected from NICU while one in twenty admissions were rejected from SCBU. The model also suggests that a marginal increase of the number of cots will be sufficient at SCBU to reduce admission rejection to less than $5 \%$, but a substantial number of extra cots is required at NICU to reduce rejection to less than $10 \%$. The proposed overflow model takes into account the absence of waiting facility in the perinatal centre and gives us an estimate of the capacity required for pre-specified performance levels. Performance was measured on the basis of the proportion of babies overflowing to temporary care and babies rejected from the perinatal centre. Using overflow and rejection probabilities, optimal cot capacity can be determined. The model derived for UCLH with two main units and TC, can easily be generalised for any other perinatal centre with three main units and TC. Although overflow from SCBU to NICU was not observed for the UCLH perinatal centre during 2006, it may occur in future years or in other centres and will be investigated in the future. The assumption of Poisson arrivals may be sometimes impractical and difficult to support. This issue will also be the focus of further research, where alternative non-Poisson arrivals will be considered. Relaxing assumptions on arrivals, LoS patterns, and flows will inevitably complicate and possibly prevent the derivation of closed form solutions using a queueing theory approach. Simulation models will be developed to cope with this increasing complexity.

Acknowledgements The authors acknowledge Dr Jane Hawdon, EGA UCL Institute of Child Health and Daniel Wood, Maternity and Neonatal Services, University College London Hospital for their help with data gathering and expert opinion. The authors also acknowledge the reviewers for their useful comments.

\section{References}

1. Abdalla N, Boucherie RJ (2002) Blocking probabilities in mobile communications networks with time-varying rates and redialing subscribers. Annals of Operations Research 112:1534 
2. Asaduzzaman M, Chaussalet TJ (2008) Modelling and performance measure of a perinatal network centre in the United Kingdom. In: Proceedings of the 21th IEEE International Symposium on Computer-Based Medical Systems, pp 506-511

3. BLISS (2005) Neonatal Services -are they improving? BLISS- The Premature Baby Charity

4. Boucherie RJ, Mandjes M (1998) Estimation of performance measures for product form cellular mobile communications networks. Telecommunication Systems 10:321-354

5. Chaussalet TJ, Xie H, Millard P (2006) A closed queueing approach to the analysis of patient flow in health care sysytems. Methods of Information in Medicine 5:492-497

6. Davis JL, Massey WA, Whitt W (1995) Sensitivity to the service-time distribution in the nonstationary Erlang loss model. Management Science 41(6):1107-1116

7. DH (2005) Report of the Neonatal Intensive Care Services Review Group. Department of Health

8. van Dijk NM (1993) Queueing Networks and Product Forms: A Systems Approach. John Wiley \& Sons, Chichester

9. Erhardsson T (2001) On the number of lost customers in stationary loss systems in the light traffic case. Queueing Systems 38(1):25-47

10. Ferreira RB, Coelli FC, Pereira WC, Almeida RM (2008) Optimizing patient flow in a large hospital surgical centre by means of discrete-event computer simulation models. Journal of Evaluation in Clinical Practice 14:1031-1037

11. Głąbowski M, Kubasik K, Stasiak M (2008) Modeling of systems with overflow multi-rate traffic. Telecommunication Systems 37:85-96

12. Griffiths JD, Price-Lloyds N, Smithies M, Williams J (2006) A queueing model of activities in an intensive care unit. IMA Journal of Management Mathematics 17(3):277-288

13. Jiang L, Giachetti RE (2008) A queueing network model to analyze the impact of parallelization of care on patient cycle time. Health Care Management Science 11:248-261

14. Kelly FP (1979) Reversibility and Stochastic Networks. John Wiley \& Sons, Chichester

15. Kelly FP (1991) Loss network. Annals of Applied Probability 1(3):319-378

16. Kim SC, Horowitz I, Young KK, Buckley TA (1999) Analysis of capacity management of the intensive care unit in a hospital. European Journal of Operational Research 115(1):3646

17. Koizumi N, Kuno E, Smith TE (2005) Modelling patient flows using a queuing network with blocking. Health Care Management Science 8(1):49-60

18. Kortbeek N, van Dijk NM (2007) On dimensioning intensive care units. AENORM 57:2226

19. Litvak N, van Rijsbergen M, Boucherie RJ, van Houdenhoven M (2008) Managing the over flow of intensive care patients. European Journal of Operational Research 185(3):9981010

20. Parmanum J, Field D, Rennie J, Steer P (2000) National census of availability of neonatal intensive care. British Medical Journal 321(7263):727-729

21. RCPCH (2007) Modelling the future: A consultation paper on the future of childrens health services. Royal College of Paediatrics and Child Health

22. Ridge J, Jones S, Nielsen M, Shahani A (1998) Capacity planning for intensive care units. European Journal of Operational Research 105(2):346-355

23. Sendfeld P (2008) Two queues with weighted one-way overflow. Methodology and Computing in Applied Probability 10(4):531-555 